\title{
Motion stability of high-speed maglev systems in consideration of aerodynamic effects: a study of a single magnetic suspension system
}

\author{
Han $W_{u^{1,2,3}} \cdot$ Xiao-Hui Zeng ${ }^{1,2}$ • Yang $\mathrm{Yu}^{3}$
}

Received: 7 February 2017 / Revised: 28 March 2017 / Accepted: 1 June 2017 / Published online: 5 August 2017

(C) The Chinese Society of Theoretical and Applied Mechanics; Institute of Mechanics, Chinese Academy of Sciences and Springer-Verlag GmbH Germany 2017

\begin{abstract}
In this study, the intrinsic mechanism of aerodynamic effects on the motion stability of a high-speed maglev system was investigated. The concept of a critical speed for maglev vehicles considering the aerodynamic effect is proposed. The study was carried out based on a single magnetic suspension system, which is convenient for proposing relevant concepts and obtaining explicit expressions. This study shows that the motion stability of the suspension system is closely related to the vehicle speed when aerodynamic effects are considered. With increases of the vehicle speed, the stability behavior of the system changes. At a certain vehicle speed, the stability of the system reaches a critical state, followed by instability. The speed corresponding to the critical state is the critical speed. Analysis reveals that when the system reaches the critical state, it takes two forms, with two critical speeds, and thus two expressions for the critical speed are obtained. The conditions of the existence of the critical speed were determined, and the effects of the control parameters and the lift coefficient on the critical speed were analyzed by numerical analysis. The results show that the first critical speed appears when the aerodynamic force is upward, and the second critical speed appears when the aerodynamic
\end{abstract}

Xiao-Hui Zeng

zxh@imech.ac.cn

1 Key Laboratory for Mechanics in Fluid Solid Coupling Systems, Institute of Mechanics, Chinese Academy of Sciences, Beijing 100190, China

2 School of Engineering Science, University of Chinese Academy of Sciences, Beijing 100049, China

3 State Key Laboratory of Hydraulic Engineering Simulation and Safety, School of Civil Engineering, Tianjin University, Tianjin 300072, China force is downward. Moreover, both critical speeds decrease with the increase of the lift coefficient.

Keywords High-speed maglev system · Critical speed . Aerodynamic effect $\cdot$ Motion stability

\section{Introduction}

In contrast to traditional wheel-rail trains, the electromagnetic suspension (EMS) maglev train does not make physical contact with the rail. EMS has attracted increasing attention due to its many advantages, such as a lower energy consumption, a smaller environmental impact, less noise, less maintenance, strong climbing ability, and small curve negotiation [1]. In China, efforts have been made toward the development of a high-speed maglev train with a speed of $600 \mathrm{~km} / \mathrm{h} \mathrm{[2]}$. Ensuring the suspension dynamic stability of EMS maglev trains is one of the key challenges in the safety of maglev transportation. In recent years, research has focused on the effects of vehicle, guideway, and control parameters on the stability of the maglev vehicle system, as well as methods for improving the dynamic stability of the system. Conditions leading to Hopf bifurcation were analyzed by $\mathrm{Wu}$ et al. [3] for a maglev control system using the test function for Hopf bifurcation. Extensive eigenvalue calculations can be avoided when analyzing the system stability. The influence of the vehicle speed and the main parameters on the dynamic stability were studied by Li et al. [4], according to the simplified kinetic model of a vehicle-guideway vibration system. The self-excited vibration mechanism of the vehicle-guideway system was explained by $\mathrm{Li}$ et al.[5] according to the energy transferred from the suspension system to the bridge, who also analyzed the influence of 
the proportional-differential (PD) controller parameters and signal delay on the stability. Numerical and experimental analysis of the influence of the natural frequency and damping of the guideway on the dynamic performance of a maglev vehicle was performed by Han et al. [6]. The effects of vehicle speed, system control parameters, and disturbance on the dynamic stability of maglev vehicles were studied by $\mathrm{Wu}$ et al. [7], who also studied the problem of the attracting domain and the chaotic dynamic characteristics of the stability control $[8,9]$. The Hopf bifurcation and resonance of a maglev system with time-delay feedback control were studied by Wang et al. [10-12] using the central manifold and the Poincaré normal form. The time delay was used as the Hopf-bifurcation condition. It was found that when the time delay exceeds a certain value, Hopf bifurcation occurs. The relationship between the periodic solution and the excitation and control parameters was also examined. The stability and the Hopf bifurcation of a rigid guideway maglev train with position-delay and speed-delay feedback and an elasticguaideway maglev train with position-delay feedback were discussed by Zhang et al. [13-15] using center manifold and normal form theory. The normal equation was obtained, and the stability of the limit cycle was determined. The stability of the three-delay state feedback control system was analyzed, and the influence of the delay and the gain of the speed feedback control on the system stability was obtained.

Many scholars have proposed novel strategies to control the coupled vibration of the vehicle and its guideway and thus improve the stability. A neuro-proportional-integral (PI) controller was developed by Yau [16] to control the dynamic response of a maglev vehicle near the permissive acceleration. The concept of a virtual-tuning mass damper was proposed by Zhou et al. [17]. An electromagnetic force was employed to simulate the force acting on the track by using a real tuned mass damper to suppress the self-excited vibration of vehicle-track system. A modified minimum mean square elimination algorithm with phase correction (C-LMS) was proposed for eliminating the self-excited vibration [18]. A method for controlling the amplitude of the vibration by adjusting the voltage was also proposed [19]. The use of a virtual electromagnet energy collector was proposed by Li et al. [20] to control the self-excited vibration of a vehicle-bridge system. Electromagnetic forces were employed to simulate the forces acting on a bridge by using a real energy harvester. Magnetic-flux feedback control was used by Li et al. [21] instead of traditional current feedback control, which simplified the interaction system. The suppression of the selfexcited vibration by adding a feedback electrical damping increment of the bridge vertical velocity was also proposed.

Previous studies on the stability of the maglev vehicle system have focused on the dynamic stability, the parameter influence, and the control of the vehicle-guideway coupling system under low- and medium-speed situations. Studies on the dynamics of high-speed maglev vehicles with the aerodynamic load effect are rare. The speed of the train operating on the Shanghai Maglev Test Line in China can reach $430 \mathrm{~km} / \mathrm{h}$, and China recently launched the research on a $600 \mathrm{~km} / \mathrm{h}$ speed maglev train. The aerodynamic load is proportional to the square of the relative velocity between vehicle and the wind [22]. An increase in the speed of the maglev train inevitably leads to the rapid increase of the aerodynamic load. The maglev vehicle does not make contact with the track during operation. Hence, the external loads of the vehicle structure are only electromagnetic and aerodynamic forces. The interaction of these two forces determines the motion characteristics of the maglev vehicle. The aerodynamic load of a maglev train at high speeds has an important effect on its dynamic stability. The aerodynamic load was found by Zeng et al. [22,23] and Li et al. [24] to have a significant impact on the dynamic characteristics of high-speed wheel-rail trains, which prompted us to study the effects of the aerodynamic load on the motion stability of high-speed maglev trains.

Some research has focused on the influence of the aerodynamic load on the forced vibration of maglev vehicles. The response of a gust-levitated maglev vehicle passing over a guide suspension bridge was simulated by Kwon et al. [25]. Their results showed that the vibration of the vehicle is sensitive to vehicle speed and wind load because of the interaction of vehicle and bridge, and that the ride comfort is reduced by the low-frequency vibration of the vehicle owing to wind turbulence. An analysis model was established by Yau [26] by simulating the guideway as a series of simply supported beams of equal length and the maglev vehicle as a rigid body. Pneumatic loads owing to unsteady airflow were considered. The response of the vehicle-guideway coupling system under a wind load was calculated. Studies show that the aerodynamic load can cause significant amplification of the acceleration of a high-speed maglev vehicle. Yau proposed a proportional-integral-differential (PID)+linear quadratic regulator (LQR) controller for reducing the acceleration response of vehicle. A 5-degree-of-freedom maglev vehicle-elastic guideway coupling model was established by Wu and Shi [27]considering the effects of the wind field. The aerodynamic characteristics of the maglev-guideway system were analyzed using a finite-element software. The RungeKutta-Merson method was used to analyze the dynamic response of the maglev vehicle under a wind field. The effects of wind speed, vehicle speed, and feedback gain coefficient were examined.

These studies contribute to our understanding of the impact of aerodynamic loads on the dynamic response of maglev trains and the stability of medium- and low-speed maglev vehicles. However, the following points are worthy of attention. (1) In the existing research, the aerodynamic load was considered as the forcing force, and attention was paid to the influence of the aerodynamic load on the forced vibration 
response of the maglev vehicle. However, the dynamic stability of the maglev system considering the aerodynamic load is a self-excited vibration problem, and the self-excited vibration stability characteristic cannot be determined by solving the forced vibration. (2) Previous studies on the dynamic stability of the maglev system focused on medium- and lowspeed vehicles, and the aerodynamic load effect was not considered. (3) The influence of the aerodynamic load on the dynamic stability of the maglev vehicle has not been studied so far. Research on the effects of the aerodynamic load and the system parameters on the dynamic stability remains insufficient. None of the previous studies considered the following problem: when the maglev train operates at a high speed, the aerodynamic load increases sharply, and the aerodynamic load changes the equilibrium position of the maglev system. This alters the inherent parameters of the magnetic levitation system, affecting its stability. When a certain vehicle speed is reached, the suspension system may become unstable. This speed is the critical speed of the high-speed maglev system and depends on the aerodynamic load.

According to the aforementioned considerations, we established a single-magnet suspension model considering the aerodynamic lift. Using this model, we analyzed the inherent effect of the aerodynamic load on the motion stability of the suspension system. The results show that the motion stability of the system changes with the increase of the vehicle speed. At a certain speed, the system reaches the critical state and becomes unstable. Thus, we propose, for the first time, the concept of the critical velocity of maglev vehicles considering aerodynamic load effects. This concept is used to analyze the motion stability of maglev trains. The two forms of the critical state are determined by using the single-magnet model, and the formula for calculating the corresponding critical speeds for two kinds of instability is derived. The conditions giving rise to the two types of instability are determined. Finally, the effects of the control parameters and the lift coefficient on the critical speed are analyzed by numerical analysis.

\section{System model}

Assume that the maglev vehicle is running on a flat and straight guideway at a constant speed. The aerodynamic load is steady and the unsteady aerodynamic load is not considered in this paper. The single-magnet suspension control model is the basic unit of the maglev vehicle and reflects the dynamic basic characteristics of the whole maglev system. To clarify the influence of the aerodynamic load on the motion stability of the maglev system, to propose relevant concepts, and to obtain explicit analytical expressions, this paper simplifies the maglev vehicle system into a single-magnet suspension model as shown in Fig. 1, and analyzes the stability mechanism of the maglev vehicle considering the aerodynamic load effect. In Fig. 1, the guideway is considered as rigid. $m$ is the mass of the electromagnet, $F$ is the electromagnetic force, $F_{\text {lift }}$ is the pneumatic lift, $Z$ is the electromagnet suspension clearance, $R$ is the electromagnet resistance, $I$ is the magnet current, $V$ is the electromagnet control voltage, $v$ is the vehicle speed, and $U$ is the oncoming wind speed.

The equation for the vertical motion of the electromagnet is

$m \ddot{Z}=m g-F+F_{\text {lift }}$.

The levitation force between the electromagnet and the guideway is calculated using the classical electromagneticforce formula [28]

$$
F(I, Z)=\frac{\mu_{0} A N^{2} I^{2}}{4 Z^{2}}
$$

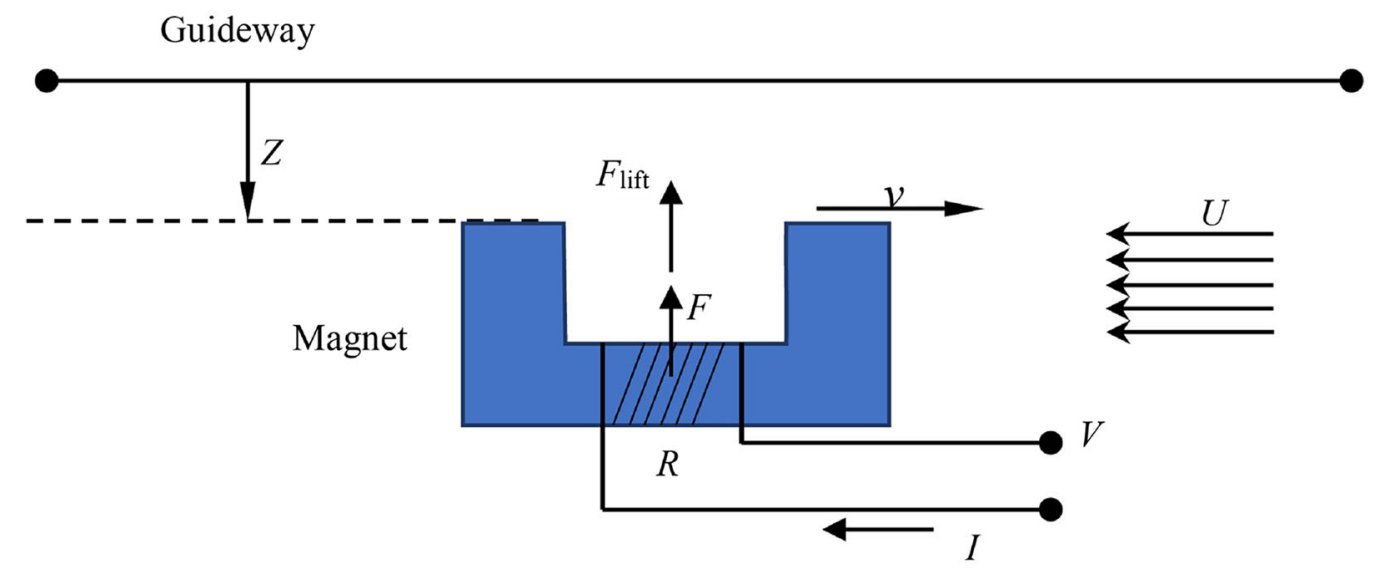

Fig. 1 Schematic of the single-magnet suspension system 
where $\mu_{0}$ is the magnetic permeability of vacuum, $Z$ is the electromagnet suspension clearance, $A$ is the effective area of the electromagnet, and $N$ is the number of coil turns. Two preconditions for using the classical electromagnetic-force formula are: (1) the suspension gap is small and uniformly distributed; (2) the magnetic reluctance and flux saturation are neglected. Because the magnetic permeability of air is equal to the magnetic permeability of a vacuum, the magnetic permeability of vacuum is adopted in Eq. (2) in place of the magnetic permeability of air.

Considering the oncoming steady air flow of the maglev train, the aerodynamic lift is constant at a certain vehicle speed and wind speed. The aerodynamic lift force can be expressed as [22,29]

$F_{\text {lift }}=\frac{1}{2} C_{\mathrm{L}} \rho A_{\mathrm{v}}(U+v)^{2}$,

where $\rho$ is the air density, $A_{\mathrm{V}}$ is the reference area of the vehicle, $C_{\mathrm{L}}$ is the aerodynamic-lift coefficient, $v$ is the vehicle speed, and $U$ is the oncoming wind speed.

According to the Faraday theorem, the governing equation of the magnetic-levitation control system is

$N \frac{\mathrm{d} \phi}{\mathrm{d} t}=-R I+V$

Equations (1)-(4) constitute the dynamic model of the singlemagnet-rigid guideway suspension system considering the aerodynamic lift.

Suppose that the equilibrium position of the stable suspension is $\left(I_{0}, \delta_{0}\right)$, where $I_{0}$ is the stable current, and $\delta_{0}$ is the stable suspension clearance. The stable voltage at the equilibrium position is $V_{0}$. Suppose that $i$ is the current disturbance at the equilibrium position, $u$ is the voltage disturbance, and $z$ is the disturbance of the vertical position of the electromagnet. To analyze the stability of a single-magnet rigid suspension system with aerodynamic lift at the equilibrium position $\left(I_{0}, \delta_{0}\right)$, the nonlinear system dynamics model was linearized at the equilibrium position. The stability of the suspension system at the equilibrium position was analyzed by evaluating the stability of the trivial solution of the linearized model. The linearization model is expressed as

$m \cdot \ddot{z}(t)=-\Delta F(i, z)$,

$\Delta F(i, z)=k_{i} \cdot i(t)-k_{c} \cdot z(t)$,

$u(t)=R \cdot i(t)+L_{0} \cdot \dot{i}(t)-k_{i} \cdot \dot{z}(t)$.

Here,

$I_{0}=\frac{2 \delta_{0}}{N} \sqrt{\frac{m g+F_{\text {lift }}}{\mu_{0} A}}, \quad k_{i}=\frac{\mu_{0} A N^{2} I_{0}}{2 \delta_{0}^{2}}$,

$k_{c}=\frac{\mu_{0} A N^{2} I_{0}^{2}}{2 \delta_{0}^{3}}, \quad L_{0}=\frac{\mu_{0} A N^{2}}{2 \delta_{0}}$.
By setting $\ddot{z}, z$, and $\dot{z}$ as the feedback-control variables, the voltage control formula under feedback is expressed as

$u=k_{\mathrm{p}} \cdot z+k_{\mathrm{d}} \cdot \dot{z}+k_{\mathrm{a}} \cdot \ddot{z}$

Here, $k_{\mathrm{p}}, k_{\mathrm{d}}$, and $k_{\mathrm{a}}$ are the feedback gains of the position, speed, and acceleration, respectively.

\section{Mechanism analysis}

The aerodynamic lift $F_{\text {lift }}$ increases sharply with increasing vehicle speed, as shown in Eq. (3). Equation (6) shows that the steady current $I_{0}$ depends on $F_{\text {lift }}$. Therefore, the aerodynamic load affects the suspension-system parameters and the effect increases with the vehicle speed. In this section, we investigate the influence mechanism of the aerodynamic lift force on movement stability, on the basis of the linear model established in Sect. 2.

Consider the feedback-control quality $x=[\ddot{z} z \dot{z}]^{\mathrm{T}}$ as the state variable. The equation for the vertical vibration of the electromagnet is

$\ddot{z}=-\frac{k_{i} i}{m}+\frac{k_{c} z}{m}$.

Then, we obtain

$$
\begin{aligned}
\dddot{z} & =-\frac{k_{i}}{m} \dot{i}+\frac{k_{c}}{m} \dot{z} \\
& =-\frac{k_{i}}{m}\left(\frac{k_{i}}{L_{0}} \cdot \dot{z}-\frac{R}{L_{0}} \cdot i+\frac{1}{L_{0}} u\right)+\frac{k_{c}}{m} \dot{z} \\
& =-\frac{R}{L_{0}} \ddot{z}+\frac{R I_{0}^{2}}{\delta_{0}^{2} m} z-\frac{I_{0}}{\delta_{0} m} u .
\end{aligned}
$$

The state equation of the system is

$$
\dot{\boldsymbol{x}}=\boldsymbol{A x}+\boldsymbol{B u},
$$$$
\boldsymbol{u}=\boldsymbol{K} \boldsymbol{x} \text {, }
$$

$\boldsymbol{A}=\left[\begin{array}{ccc}-\frac{R}{L_{0}} & \frac{R I_{0}^{2}}{\delta_{0}^{2} m} & 0 \\ 0 & 0 & 1 \\ 1 & 0 & 0\end{array}\right], \quad \boldsymbol{B}=\left[\begin{array}{c}-\frac{I_{0}}{\delta_{0} m} \\ 0 \\ 0\end{array}\right]$

$\boldsymbol{K}=\left[k_{\mathrm{a}} k_{\mathrm{p}} k_{\mathrm{d}}\right]$.

According to the state feedback-control law, the characteristic matrix of the closed-loop system is

$\boldsymbol{A}+\boldsymbol{B} \cdot \boldsymbol{K}=\left[\begin{array}{ccc}-\frac{R}{L_{0}}-\frac{I_{0}}{\delta_{0} m} k_{\mathrm{a}} & \frac{R I_{0}^{2}}{\delta_{0}^{2} m}-\frac{I_{0}}{\delta_{0} m} k_{\mathrm{p}} & -\frac{I_{0}}{\delta_{0} m} k_{\mathrm{d}} \\ 0 & 0 & 1 \\ 1 & 0 & 0\end{array}\right]$ 
The characteristic equation is

$\lambda^{3}+a_{1} \lambda^{2}+a_{2} \lambda+a_{3}=0$.

Here, the parameters are given as

$a_{1}=\frac{R}{L_{0}}+\frac{I_{0}}{\delta_{0} m} k_{\mathrm{a}}, \quad a_{2}=\frac{I_{0}}{\delta_{0} m} k_{\mathrm{d}}$,

$a_{3}=-\frac{R I_{0}^{2}}{\delta_{0}^{2} m}+\frac{I_{0}}{\delta_{0} m} k_{\mathrm{p}}, \quad I_{0}=\frac{2 \delta_{0}}{N} \sqrt{\frac{m g+F_{\text {lift }}}{\mu_{0} A}}$.

The stability condition of the trivial solution in Eq. (10) is that the real parts of the three characteristic value roots are less than zero. According to the Routh-Hurwitz criterion, the necessary and sufficient condition for the real part of the eigenvalue to be negative is

$a_{1}>0, \quad a_{1} a_{2}>a_{3}, \quad a_{3}>0$.

The range of the control parameters can be determined by Eq. (16) is shown in Eq. (17). If the aerodynamic lift changes, the range of the control parameters also changes.

$k_{\mathrm{p}}>\frac{R I_{0}}{\delta_{0}}, \quad k_{\mathrm{a}}>-\frac{R \delta_{0} m}{L_{0} I_{0}}, \quad k_{\mathrm{d}}>\frac{\left(k_{\mathrm{p}} \delta_{0}-R I_{0}\right) L_{0} m}{R \delta_{0} m+k_{\mathrm{a}} I_{0} L_{0}}$.

If the feedback-control parameter $\left[k_{\mathrm{a}} k_{\mathrm{p}} k_{\mathrm{d}}\right]$ is fixed, the electromagnet stable suspension clearance $\delta_{0}$ remains unchanged. According to Eq. (6), the equilibrium current $I_{0}$ changes because of the aerodynamic lift force, which alters the equilibrium position of the system. The linearization system represented by Eq. (5) depends on the equilibrium position $\left(I_{0}, \delta_{0}\right)$. The alteration of the equilibrium position changes the characteristic matrix (13) of the closed-loop system and the coefficients of the characteristic equation (14). Thus, the aerodynamic lift influences the motion stability of the suspension system.

As the speed increases, the aerodynamic lift increases. Naturally, the equilibrium position $\left(I_{0}, \delta_{0}\right)$ varies with the vehicle speed. This means that the stability of a rigidguideway single-magnet suspension system is independent of the vehicle speed if the aerodynamic lift is not considered. If the aerodynamic lift is considered, the motion stability of the suspension system is related to the vehicle speed. If the aerodynamic lift is not considered or is zero, the selected control parameters $\left[k_{\mathrm{a}} k_{\mathrm{p}} k_{\mathrm{d}}\right]$ satisfy the stability condition of Eq. (17). However, as the speed increases and the aerodynamic lift exceeds a certain value, the control parameters do not satisfy the stability condition. Thus, bifurcation and instability of the suspension system may occur.

According to the aforementioned analysis, we propose the novel concept of a critical speed for examining the motion stability of the maglev train considering aerodynamic effects. Under certain aerodynamic conditions, when the vehicle speed exceeds a specific value, the real parts of the eigenvalue of the characteristic matrix are equal to zero, the suspension system reaches the critical state and a bifurcation occurs. This speed is called the critical speed.

The necessary and sufficient condition for the asymptotic stability of the trivial solution of Eq. (10) is that the real part of the eigenvalue is less than zero. In the critical state, the real part of the eigenvalue is equal to zero. Suppose that the complex number $\lambda=\alpha+\mathrm{i} \beta$ (where $\alpha$ and $\beta$ are real numbers) is the root of the characteristic equation (14). It is substituted into the characteristic equation, and the real and imaginary parts are separated. Then, we obtain

$$
\begin{aligned}
& \alpha^{3}-3 \alpha \beta^{2}+a_{1} \alpha^{2}-a_{1} \beta^{2}+a_{2} \alpha+a_{3}=0 \\
& 3 \alpha^{2} \beta-\beta^{3}+2 a_{1} \alpha \beta+a_{2} \beta=0
\end{aligned}
$$

When the real part of the eigenvalue $\lambda$ is equal to zero, the stability of the maglev system at the equilibrium position is in the critical state. The three characteristic roots of the characteristic equation (14) comprise a pair of conjugate complex eigenvalues and a real eigenvalue. The system stability has the following two critical states.

(1) The first kind of critical state: the real parts of the pair of conjugate eigenvalue are equal to zero. Thus, $\alpha=0, \lambda=$ $\pm \mathrm{i} \beta$, and a dynamic bifurcation (Hopf bifurcation) occurs in the suspension system. This results in the maglev system having a motion of limited cycle under disturbance instead of decaying to zero. Accordingly, Eq. (18) becomes

$-a_{1} \beta^{2}+a_{3}=0$, $a_{2} \beta-\beta^{3}=0$.

Then, we obtain

$a_{1} a_{2}=a_{3}$.

The critical speed is derived and expressed as Eq. (21). This is called the critical speed $A$.

$v_{\text {critical }-A}=\sqrt{\frac{\frac{\mu_{0} A N^{2} m^{2}\left(k_{\mathrm{p}}-R k_{\mathrm{d}} / L_{0}\right)^{2}}{4\left(m R+k_{\mathrm{a}} k_{\mathrm{d}}\right)^{2}}-m g}{\frac{1}{2} C_{\mathrm{L}} \rho A_{\mathrm{V}}}}-U$.

(2) The second kind of critical state: the real eigenvalue is equal to zero, i.e. $\alpha=\beta=0, \lambda=0$. Thus, a static bifurcation occurs, resulting in the increase of the electromagnet displacement in an exponential manner and the instability of the system. Accordingly, Eq. (18) becomes $a_{3}=0$. The derived critical speed for this case is shown in Eq. (22) and is denoted as $B$. 
$v_{\text {critical-B }}=\sqrt{\frac{\left(\frac{k_{\mathrm{p}}}{R}\right)^{2} \frac{\mu_{0} A N^{2}}{4 \delta_{0}^{2}}-m g}{\frac{1}{2} C_{\mathrm{L}} \rho A_{\mathrm{V}}}}-U$.

The aforementioned analysis indicates that at a certain vehicle speed, the aerodynamic loads cause the real part of the eigenvalues of the characteristic matrix to be zero and reach the critical state. This speed is called the critical speed. The critical speed has often been used in the stability analysis of wheel-rail vehicle system dynamics [30-33]. The analysis in this section shows that critical speed problem should also be considered in the motion stability analysis of a maglev system with aerodynamic loads. If the single-magnet rigid guideway suspension system given by Eqs. (1)-(4) does not consider the aerodynamic load or if the vehicle speed is zero, the system is stable at the equilibrium position. When the speed is less than the critical speed, the system is stable. When the speed is greater than the critical speed, the system is unstable. When the vehicle speed is equal to the critical speed, a system bifurcation occurs in the critical state. In the next section, the expressions for the two kinds of critical speed corresponding to the two critical states of a single-magnet suspension control system are derived considering the aerodynamic lift.

\section{Numerical analysis}

To analyze the stability of the suspension system, we use the electromagnet parameters shown in Table 1. The aerodynamic load is calculated using Eq. (3). To calculate the aerodynamic load on the electromagnet, the relevant parameters for the calculation of the aerodynamic loads should be set. At present, there are no aerodynamic data for maglev trains. In this study, the aerodynamic lift of the maglev vehicle was calculated by employing the parameters used in the aerodynamic analysis of high-speed wheel-rail trains. We set the reference area as $A_{\mathrm{v}}=10 \mathrm{~m}^{2}$ and the air density as $\rho=1.225 \mathrm{~kg} / \mathrm{m}^{3}$ and assumed a wind speed $U$ of $10.7 \mathrm{~m} / \mathrm{s}$. The aerodynamic lift was divided into upward aerodynamic lift (lift coefficient is negative) and downward aerodynamic lift (lift coefficient is positive). The lift coefficients do not vary with respect to the vehicle speed.

The feedback control parameters are set as $k_{\mathrm{p}}=20,000$, $k_{\mathrm{d}}=500 . k_{\mathrm{a}}=50$ and $k_{\mathrm{a}}=60$ are selected as two cases. The selected feedback control parameters meet the stability condition shown in Eq. (17) when the aerodynamic load

Table 1 Electromagnet parameters [34]

\begin{tabular}{llllll}
\hline$\mu_{0}\left(\mathrm{~N} / \mathrm{A}^{2}\right)$ & $\delta_{0}(\mathrm{~m})$ & $N_{m}$ & $R(\Omega)$ & $A_{m}\left(\mathrm{~m}^{2}\right)$ & $m(\mathrm{~kg})$ \\
\hline $4 \pi \times 10^{-7}$ & 0.01 & 320 & 10.0 & 2.0 & $2.1 \times 10^{4}$ \\
\hline
\end{tabular}

is not considered. The conjugate eigenvalues and the real eigenvalue are calculated with respect to the vehicle speed for cases where the aerodynamic force is vertically upward and vertically downward. The critical speed is determined, and the effects of the parameters are analyzed.

\subsection{Upward aerodynamic force}

Figures 2-5 show the variation of the real part of the conjugate eigenvalue and the real eigenvalue with respect to the vehicle speed for lift coefficients of $0.01,0.02,0.03,0.04$, $0.05,0.06$. Figures 2 and 3 show the conjugate eigenvalue results for $k_{\mathrm{a}}=50$ and $k_{\mathrm{a}}=60$, respectively. Figures 4 and 5 show the real eigenvalue results for $k_{\mathrm{a}}=50$ and $k_{\mathrm{a}}=60$, respectively.

Figures 2 and 3 show that when the aerodynamic force is upward, the real part of the conjugate eigenvalue increases

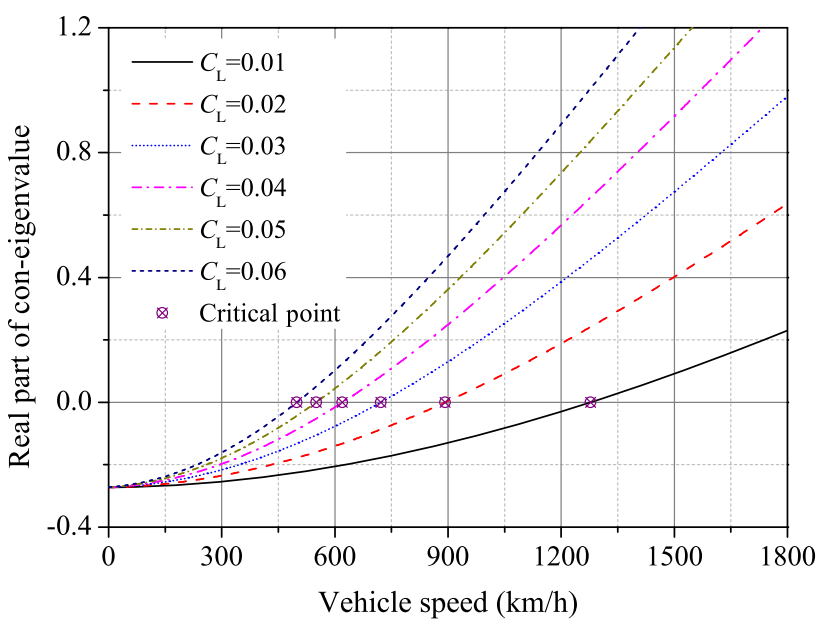

Fig. 2 Changing curve of the real part of the conjugate eigenvalue (upward aerodynamic force, $k_{\mathrm{p}}=20,000, k_{\mathrm{d}}=500$, and $k_{\mathrm{a}}=50$ )

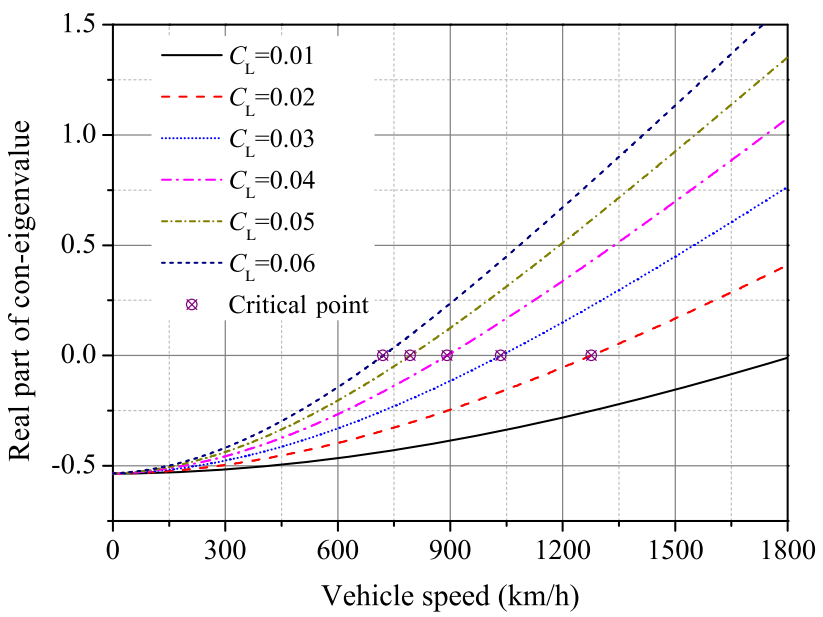

Fig. 3 Changing curve of the real part of the conjugate eigenvalue (upward aerodynamic force, $k_{\mathrm{p}}=20,000, k_{\mathrm{d}}=500$, and $k_{\mathrm{a}}=60$ ) 


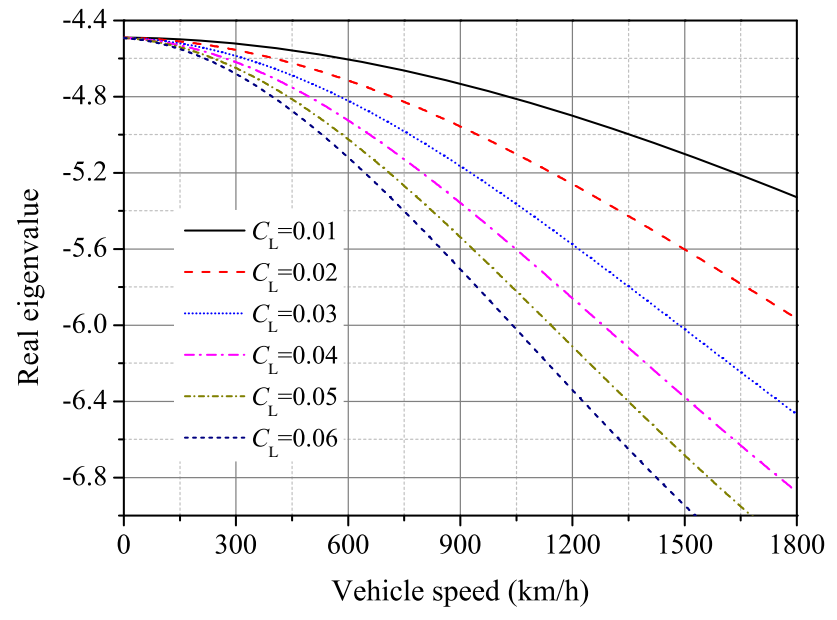

Fig. 4 Changing curve of the real eigenvalue (upward aerodynamic force, $k_{\mathrm{p}}=20,000, k_{\mathrm{d}}=500$, and $k_{\mathrm{a}}=50$ )

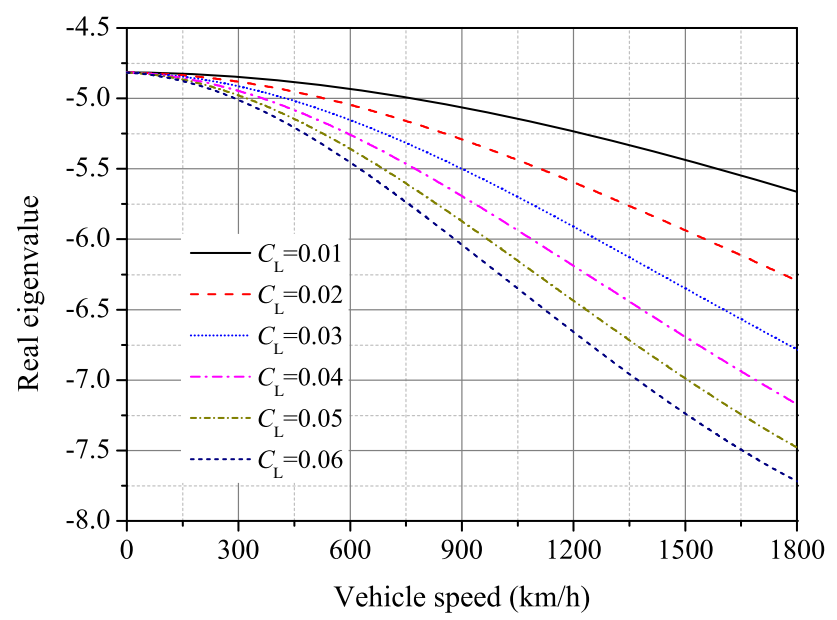

Fig. 5 Changing curve of the real eigenvalue (upward aerodynamic force, $k_{\mathrm{p}}=20,000, k_{\mathrm{d}}=500$, and $k_{\mathrm{a}}=60$ )

Table 2 Statistical table for the critical speed $A$ with an upward aerodynamic force

\begin{tabular}{llrrrrr}
\hline Lift coefficient & 0.01 & \multicolumn{1}{c}{0.02} & \multicolumn{1}{c}{0.03} & 0.04 & 0.05 & 0.06 \\
\hline$k_{\mathrm{a}}=50$ & 1278.2 & 892.5 & 721.7 & 619.8 & 550.3 & 499.0 \\
$k_{\mathrm{a}}=60$ & 1821.0 & 1276.3 & 1035.1 & 891.2 & 793.1 & 720.6 \\
\hline
\end{tabular}

with the vehicle speed. It passes through the horizontal zero line; that is, the conjugate eigenvalue crosses the imaginary axis. Then, the suspension system reaches the first kind of critical state, at the critical speed $A$. The value of critical speed $A$ shown in the figures is the same as that calculated using Eq. (21). The critical speed results shown in the two graphs are presented in Table 2.

Figures 4 and 5 show that when the aerodynamic force is upward, the real eigenvalue gradually decreases with the

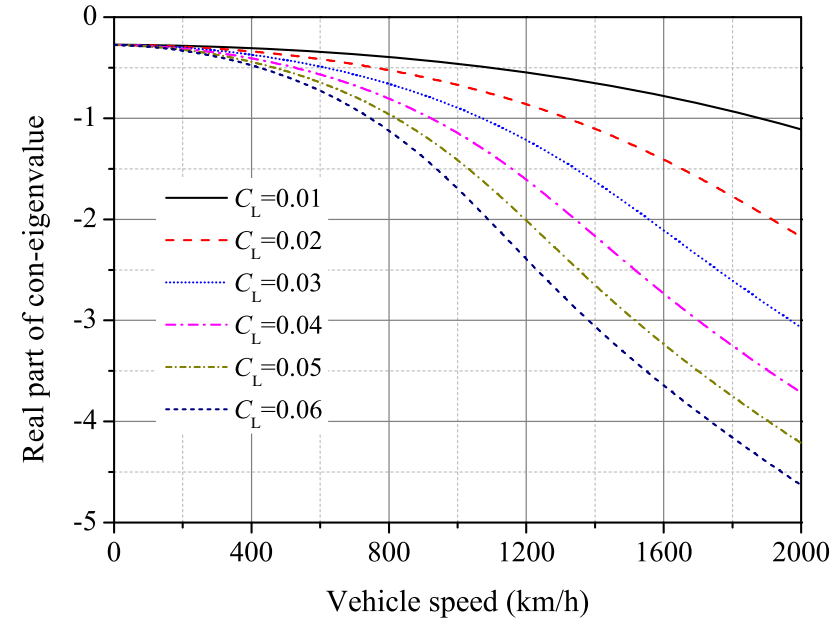

Fig. 6 Changing curve of the real part of the conjugate eigenvalue (downward aerodynamic force, $k_{\mathrm{p}}=20,000, k_{\mathrm{d}}=500$, and $k_{\mathrm{a}}=50$ )

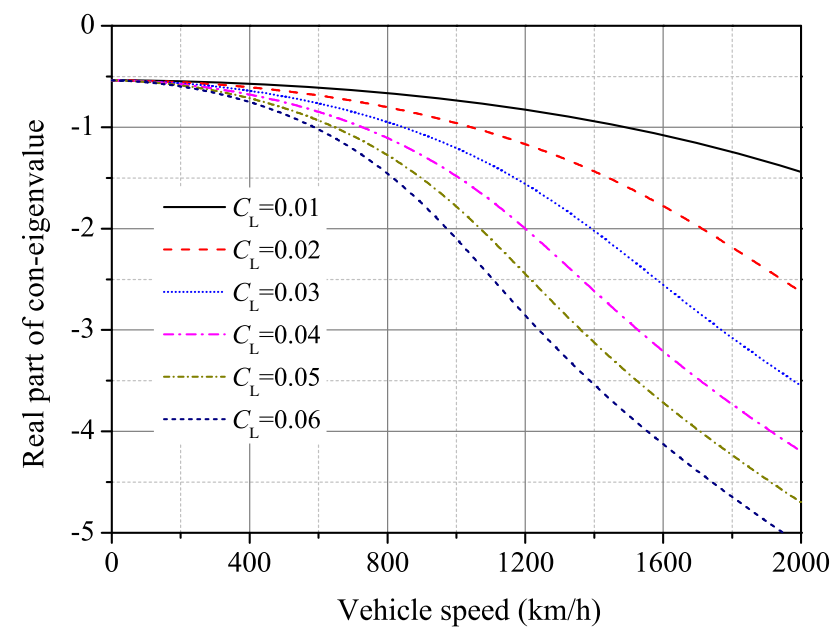

Fig. 7 Changing curve of the real part of the conjugate eigenvalue (downward aerodynamic force, $k_{\mathrm{p}}=20,000, k_{\mathrm{d}}=500$, and $k_{\mathrm{a}}=60$ )

vehicle speed. It does not intersect with the zero horizontal line. Thus, the suspension system never reaches the second kind of critical state, and the vehicle does not reach the critical speed $B$. In this case, the expression under the square root in Eq. (22) is negative; thus, the critical speed $B$ cannot be calculated.

\subsection{Downward aerodynamic force}

Figures 6-9 show the variation of the real part of the conjugate eigenvalue and the real eigenvalue with respect to the vehicle speed for lift coefficients of $0.01,0.02,0.03,0.04$, $0.05,0.06$. Figures 6 and 7 show the conjugate eigenvalue results for $k_{\mathrm{a}}=50$ and $k_{\mathrm{a}}=60$, respectively. Figures 8 and 9 show the real eigenvalues results for $k_{\mathrm{a}}=50$ and $k_{\mathrm{a}}=60$, respectively. 


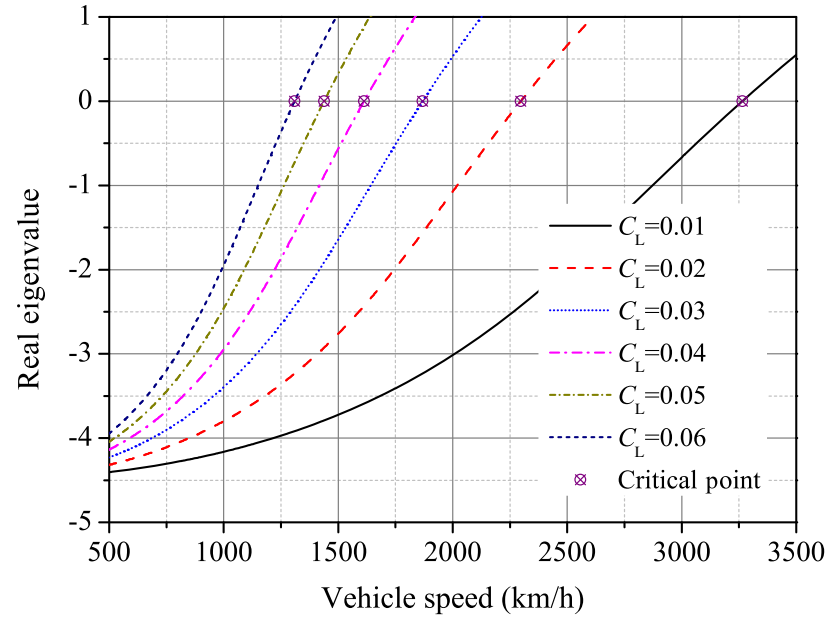

Fig. 8 Changing curve of the real eigenvalue (downward aerodynamic force, $k_{\mathrm{p}}=20,000, k_{\mathrm{d}}=500$, and $k_{\mathrm{a}}=50$ )

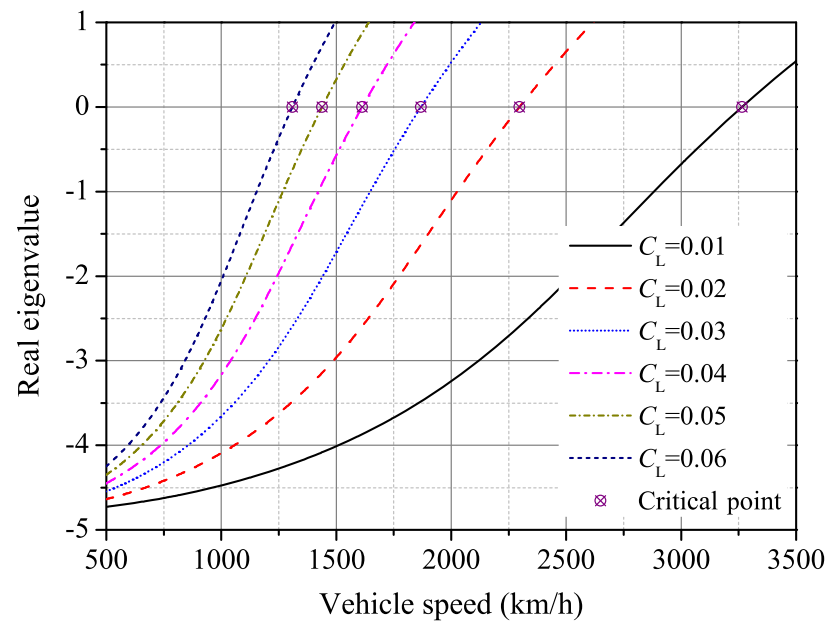

Fig. 9 Changing curve of the real eigenvalue (downward aerodynamic force, $k_{\mathrm{p}}=20,000, k_{\mathrm{d}}=500$, and $k_{\mathrm{a}}=60$ )

Figures 6 and 7 show that when the aerodynamic force is downward, the real part of the conjugate eigenvalue gradually decreases with the increase of the vehicle speed. It does not intersect with the zero horizontal line. Thus, the suspension system never reaches the first critical state, and the vehicle speed does not reach critical speed $A$. In this case, the expression under the square root in Eq. (21) is negative, and the critical speed $A$ cannot be calculated.

Unlike the results presented in Sect. 4.1, when the aerodynamic force is downward, with the increase of the vehicle speed, the real eigenvalue gradually increases and intersects with the zero horizontal line, as shown in Figs. 8 and 9. When the vehicle speed is increased until the real eigenvalue is zero, the suspension system reaches the second kind of critical state, and the vehicle speed reaches the critical speed $B$. The critical speed results shown in Figs. 8 and 9 are presented in Table 3 and are consistent with Eq. (22). In Table 3, the
Table 3 Statistical table for the critical speed $B$ with a downward aerodynamic force

\begin{tabular}{lllllll}
\hline Lift coefficient & 0.01 & 0.02 & 0.03 & 0.04 & 0.05 & 0.06 \\
\hline$k_{\mathrm{a}}=50$ & 3264.4 & 2297.0 & 1868.4 & 1613.0 & 1438.6 & 1309.9 \\
$k_{\mathrm{a}}=60$ & 3264.4 & 2297.0 & 1868.4 & 1613.0 & 1438.6 & 1309.9 \\
\hline
\end{tabular}

critical speed is equal for $k_{\mathrm{a}}=50$ and $k_{\mathrm{a}}=60$. According to Eq. (22), we also know that the critical speed $B$ is independent of the acceleration-control parameter $k_{\mathrm{a}}$ and the speed-control parameter $k_{\mathrm{d}}$.

The data in Tables 2 and 3 show that a higher aerodynamic coefficient yields a lower critical velocity, regardless of whether the aerodynamic lift is upward or downward.

\subsection{Condition for the existence of the critical speed}

As discussed in Sect. 4.1 and 4.2, when the aerodynamic force is downward, the critical speed $A$ does not exist. In this case, the expression under the square root in Eq. (21) is negative, and the critical speed cannot be calculated. When the aerodynamic force is upward, the critical speed $B$ does not exist. In this case, the expression under the square root in Eq. (21) is negative, and the critical speed cannot be calculated. Thus, we deduce the following.

(1) The expression under the square root in Eq. (21) being greater than zero is a necessary condition for the vehicle reaching the critical speed $A$, i.e.

$\frac{\frac{\mu_{0} A N^{2} m^{2}\left(k_{\mathrm{p}}-R k_{\mathrm{d}} / L_{0}\right)^{2}}{4\left(m R+k_{\mathrm{a}} k_{\mathrm{d}}\right)^{2}}-m g}{\frac{1}{2} C_{\mathrm{L}} \rho A_{\mathrm{V}}}>0$.

If the single-magnet-rigid guideway suspension system given by Eqs. (1)-(4) considering the aerodynamic lift satisfies the conditions of Eq. (23) and the aerodynamic load is not considered or the vehicle speed is zero, the suspension system is stable at the equilibrium position. The stability of the system at the equilibrium position is described as follows. When $v<v_{\text {critical }-A}$, the system is stable. When $v>v_{\text {critical }-A}$, the system is disturbed and no longer convergent, and the cycle motion is limited. When $v=v_{\text {critical }-A}$, Hopf bifurcation occurs in the system, and the system is in the critical state. (2) The necessary condition for the vehicle to reach the critical speed $B$ is that the expression under the square root in Eq. (22) is greater than zero, i.e.

$$
\frac{\left(\frac{k_{\mathrm{p}}}{R}\right)^{2} \frac{\mu_{0} A N^{2}}{4 \delta_{0}^{2}}-m g}{\frac{1}{2} C_{\mathrm{L}} \rho A_{\mathrm{v}}}>0 .
$$




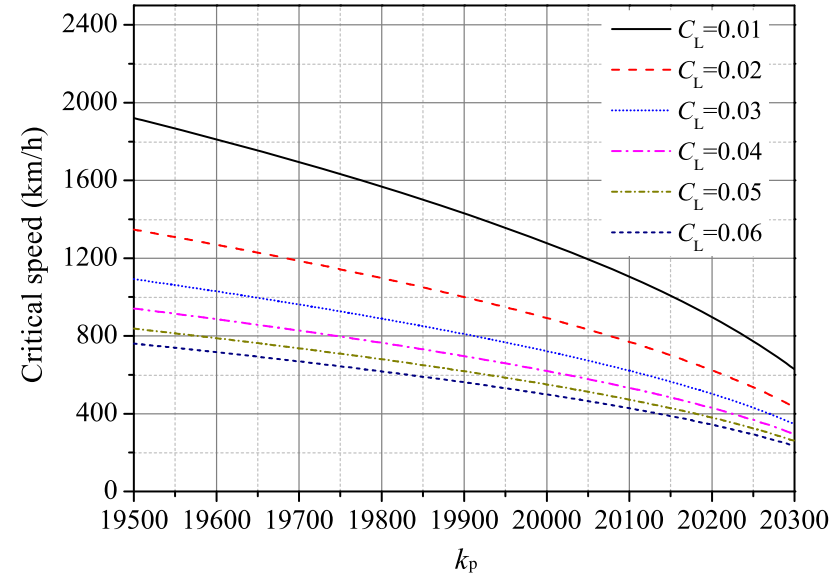

Fig. 10 Relationship between the control parameter $k_{\mathrm{p}}$ and the critical speed $A$ (upward aerodynamic force, $k_{\mathrm{d}}=500, k_{\mathrm{a}}=50$ )

If the single-magnet rigid guideway suspension system given by Eqs. (1)-(4) considering the aerodynamic lift satisfies the conditions of Eq. (24) and the aerodynamic load is not considered or the vehicle speed is zero, the suspension system is stable at the equilibrium position. The stability of the system at the equilibrium position is described as follows. When $v<v_{\text {critical }-B}$, the system is stable. When $v>v_{\text {critical }-B}$, the displacement index of the electromagnet increases, and the system rapidly becomes unstable. When $v=v_{\text {critical }-B}$, static bifurcation occurs in the system, and the system is in the critical state.

\subsection{Relationship between critical speed and control parameters}

Figures $10-12$ show the relationship between the critical speed $A$ and the control parameters $k_{\mathrm{p}}, k_{\mathrm{d}}$, and $k_{\mathrm{a}}$ for an upward aerodynamic force. The results indicate that the critical speed $A$ decreases with the increase of $k_{\mathrm{p}}$ and increases with the increase of $k_{\mathrm{d}}$ and $k_{\mathrm{a}}$. Figure 13 shows the relationship between the critical speed $B$ and the control parameter $k_{\mathrm{p}}$ for a downward aerodynamic force. The results indicate that the critical speed $B$ increases with $k_{\mathrm{p}}$.

\section{Conclusions}

In order to facilitate proposing relevant concepts and obtaining explicit expressions, a single magnetic suspension system is adopted in the paper. The intrinsic mechanism of the aerodynamic effect on the motion stability of high-speed maglev system was revealed based on the single magnetic suspension system. The concept of the critical speed for the maglev vehicle considering aerodynamic effect was proposed. Also, the formula for calculating the critical speed was derived.

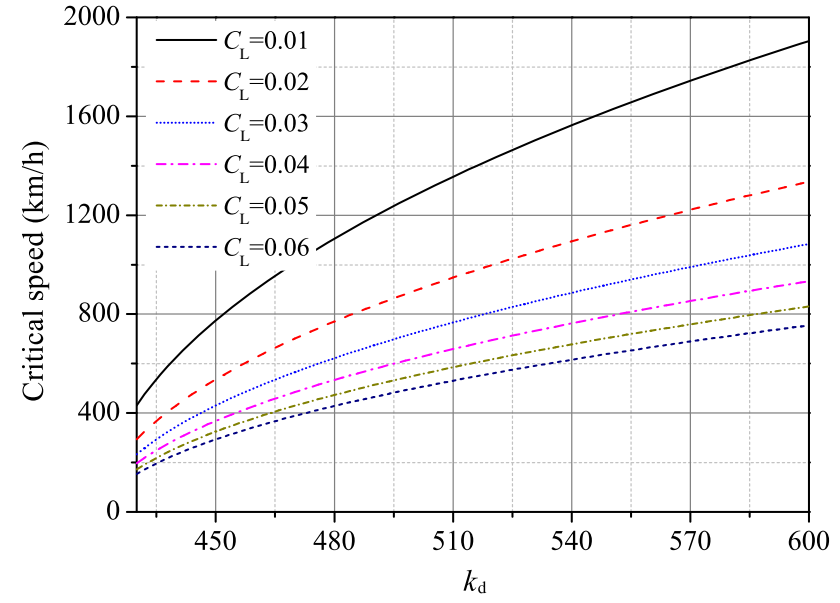

Fig. 11 Relationship between the control parameter $k_{\mathrm{d}}$ and the critical speed $A$ (upward aerodynamic force, $k_{\mathrm{p}}=20,000, k_{\mathrm{a}}=50$ )

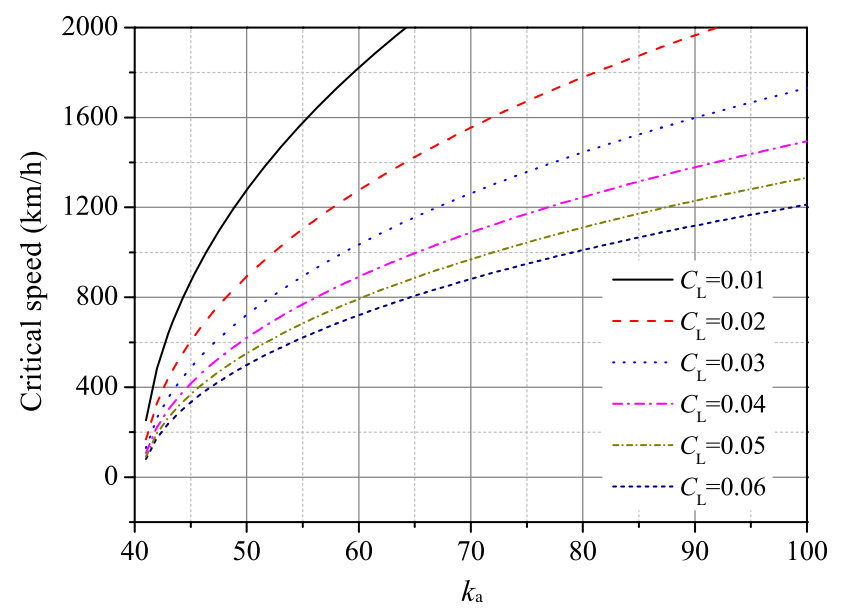

Fig. 12 Relationship between the control parameter $k_{\mathrm{a}}$ and the critical speed $A$ (upward aerodynamic force, $k_{\mathrm{p}}=20,000, k_{\mathrm{d}}=500$ )

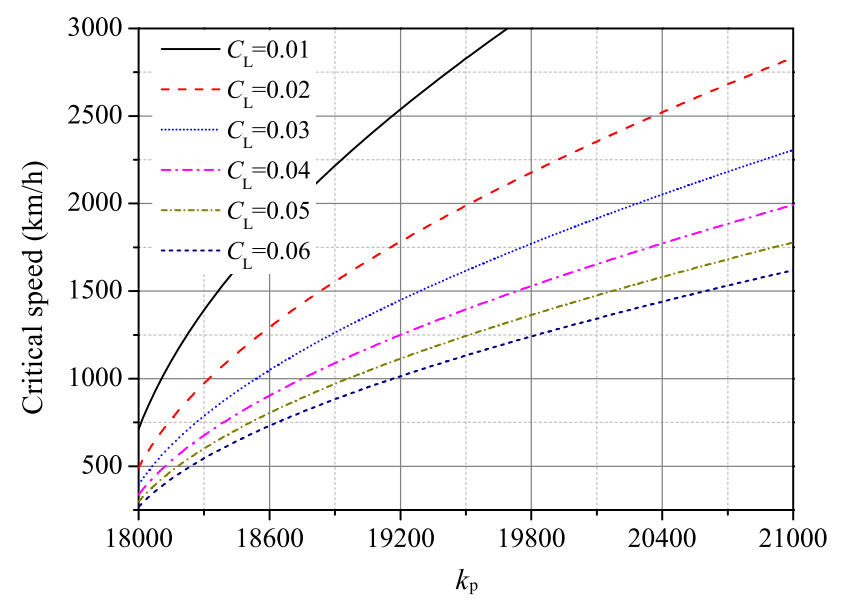

Fig. 13 Relationship between the control parameter $k_{\mathrm{p}}$ and the critical speed $B$ (downward aerodynamic force, $k_{\mathrm{d}}=500, k_{\mathrm{a}}=50$ ) 
Aerodynamic load changes the equilibrium position of the maglev-vehicle suspension system. Thus, the inherent parameters of the maglev dynamical system change, and the motion stability is affected. In this study, the motion stability of a single-magnet suspension system considering the aerodynamic lift was analyzed. The results indicate that when the speed reaches a certain value, the motion stability of the maglev system reaches a critical state because of the aerodynamic lift increasing significantly with the vehicle speed. There are two cases for reaching the critical state, and the speed corresponding to the critical state is the critical speed. The critical speed formula for the two critical states and the necessary conditions for reaching these two states were determined.

According to the concept and formula of the critical speed proposed for the high-speed maglev vehicle, a numerical analysis was performed for the motion stability of the maglev system with varying parameters. The results showed that when the aerodynamic force is upward, the first kind of instability occurs as the speed increases. In this case, the critical speed $A$ decreases with the increase of $k_{\mathrm{p}}$ and increases with the increase of $k_{\mathrm{d}}$ and $k_{\mathrm{a}}$. When the aerodynamic force is downward, the second kind of instability occurs as the speed increases. In this case, the critical speed $B$ increases with the increase of $k_{\mathrm{p}}$ and is unaffected by variations in $k_{\mathrm{d}}$ and $k_{\mathrm{a}}$. When the lift coefficient increases, the critical speeds $A$ and $B$ both decrease.

In this study, the novel concept of the critical speed for the motion stability of a high-speed maglev vehicle considering the aerodynamic load was proposed, which has potential for wide applications in the dynamic analysis and optimization design of high-speed maglev vehicles.

Acknowledgements The project was supported by the National Key Research and Development Program of China (Grant 2016YFB1200602), the National Natural Science Foundation of China (Grants 11672306, 51490673), the Strategic Priority Research Program of the Chinese Academy of Sciences (Grant XDB22020101), the National Basic Research Program (973 Program) of China (Grant 2014CB046801), and the State Key Laboratory of Hydraulic Engineering Simulation and Safety (Tianjin University).

\section{References}

1. Lee, H.W., Kim, K.C., Lee, J.: Review of maglev train technologies. IEEE Trans. Magn. 42, 1917-1925 (2006)

2. Dai, G.C.: $600 \mathrm{~km}$ high-speed maglev project started in China: build a test line within 5 years. The Study News [2016.11.13]. http://www.thestudy.cn/newsDetail_forward_1557972

3. Wu, J.J., Shen, F., Shi, X.H.: Stability and hopf bifurcation of maglev control system. J. Vib. Shock 29, 193-196 (2010). (in Chinese)

4. Li, S.Q., Zhang, K.L., Chen, Y., et al.: Judgment method of maglev vehicle dynamic stability of flexible track. J. Traffic Transp. Eng. 15, 43-49 (2015). (in Chinese)
5. Li, J.H., Li, J., Zhou, D.F., et al.: Self-excited vibration problems of maglev vehicle-bridge interaction system. J. Cent. South Univ. 21, 4184-4192 (2014)

6. Han, H.S., Yim, B.H., Lee, J.K., et al.: Effects of guideway's vibration characteristics on the dynamics of a maglev vehicle. Veh. Syst. Dyn. 47, 309-324 (2009)

7. Wu, J.J., Zheng, X.J., Zhou, Y.H., et al.: The nonlinear dynamic characteristics of an EMS maglev control system with two-stage suspension. Acta Mech. Solida Sin. 24, 68-74 (2003). (in Chinese)

8. Zheng, X.J., Wu, J.J., Zhou, Y.H.: Numerical analyses on dynamic control of five-degree-of-freedom maglev vehicle moving on flexible guideways. J. Sound Vib. 235, 43-61 (2000)

9. Zheng, X.J., Wu, J.J., Zhou, Y.H.: Effect of spring non-linearity on dynamic stability of a controlled maglev vehicle and its guideway system. J. Sound Vib. 279, 201-215 (2005)

10. Wang, H.P., Li, J., Zhang, K.: Stability and Hopf bifurcation of the maglev system with delayed speed feedback control. Acta Autom. Sin. 33, 829-834 (2007)

11. Wang, H.P., Li, J., Zhang, K.: Non-resonant response, bifurcation and oscillation suppression of a non-autonomous system with delayed position feedback control. Nonlinear Dyn. 51, 447-464 (2008)

12. Wang, H.P., Li, J., Zhang, K.: Sup-resonant response of a nonautonomous maglev system with delayed acceleration feedback control. IEEE Trans. Magn. 44, 2338-2350 (2008)

13. Zhang, L.L., Campbell, S.A., Huang, L.H.: Nonlinear analysis of a maglev system with time-delayed feedback control. Phys. DNonlinear Phenom. 240, 1761-1770 (2011)

14. Zhang, L.L., Huang, L.H., Zhang, Z.Z.: Hopf bifurcation of the maglev time-delay feedback system via pseudo-oscillator analysis. Math. Comput. Modell. 52, 667-673 (2010)

15. Zhang, L.L., Huang, L.H., Zhang, Z.Z.: Stability and Hopf bifurcation of the maglev system with delayed position and speed feedback control. Nonlinear Dyn. 57, 197-207 (2009)

16. Yau, J.D.: Vibration control of maglev vehicles traveling over a flexible guide way. J. Sound Vib. 321, 184-200 (2009)

17. Zhou, D.F., Hansen, C.H., Li, J.: Suppression of maglev vehiclegirder self-excited vibration using a virtual tuned mass damper. J. Sound Vib. 330, 883-901 (2011)

18. Zhou, D.F., Li, J., Hansen, C.H.: Application of least mean square algorithm to suppression of maglev track-induced self-excited vibration. J. Sound Vib. 330, 5791-5811 (2011)

19. Zhou, D.F., Li, J., Zhang, K.: Amplitude control of the trackinduced self-excited vibration for a maglev system. ISA Trans. 53, 1463-1469 (2014)

20. Li, J.H., Li, J., Zhou, D.F., et al.: The active control of maglev stationary self-excited vibration with a virtual energy harvester. IEEE Trans. Ind. Electron. 62, 2942-2951 (2015)

21. Li, J.H., Fang, D., Zhang, D., et al.: A practical control strategy for the maglev self-excited resonance suppression. Math. Problems Eng. 2016, 1-9 (2016)

22. Zeng, X.H., Wu, H., Lai, J., et al.: Influences of aerodynamic loads on hunting stability of high-speed railway vehicles and parameter studies. Acta. Mech. Sin. 30, 889-900 (2014)

23. Zeng, X.H., Wu, H., Lai, J., et al.: Hunting stability of high-speed railway vehicles on a curved track considering the effects of steady aerodynamic loads. J. Vib. Control 22, 4159-4175 (2016)

24. Li, S.Y., Zheng, Z.J., Yu, J.L., et al.: Dynamic simulation and safety evaluation of high-speed trains meeting in open air. Acta. Mech. Sin. 32, 206-214 (2016)

25. Kwon, S.D., Lee, J.S., Moon, J.W., et al.: Dynamic interaction analysis of urban transit maglev vehicle and guideway suspension bridge subjected to gusty wind. Eng. Struct. 30, 3445-3456 (2008)

26. Yau, J.D.: Aerodynamic vibrations of a maglev vehicle running on flexible guide ways under oncoming wind actions. J. Sound Vib. 329, 1743-1759 (2010) 
27. Wu, J.J., Shi, X.H.: Numerical analyses of dynamic stability of maglev vehicles in crosswind field. J. Lanzhou Univ. (Nat. Sci.) 45, 96-102 (2009). (in Chinese)

28. Moon, F.C.: Magneto-Solid Mechanics. Wiley, New York (1984)

29. Tian, H.Q.: Train Aerodynamics. China Railway Publishing House, Beijing (2007). (in Chinese)

30. Zeng, X.H., Wu, H., Lai, J., et al.: The effect of wheel set gyroscopic action on the hunting stability of high-speed trains. Veh. Syst. Dyn. 55, 924-944 (2017)

31. Cheng, Y.C.: Hunting stability analysis of a railway vehicle system using a novel non-linear creep model. Proc. Inst. Mech. Eng. Part F. J. Rail Rapid Transit 226, 612-629 (2012)
32. True, H.: Multiple attractors and critical parameters and how to find them numerically: the right, the wrong and the gambling way. Veh. Syst. Dyn. 51, 443-459 (2013)

33. Iwnicki, S.: Handbook of Railway Vehicle Dynamics. CRC Press, London (2006)

34. Wu, J.J., Yang, W.W.: Dynamic character of EMS maglev train/nonlinear elastic guideway system. J. Lanzhou Univ. (Nat. Sci.) 42, 120-126 (2006). (in Chinese) 